\title{
Impact of intermediate and high energy nuclear data on the neutronic safety parameters of MYRRHA accelerator driven system
}

\author{
Alexey Stankovskiya, Yurdunaz Çelik, and Gert Van den Eynde \\ SCK•CEN, Boeretang 200, 2400 Mol, Belgium
}

\begin{abstract}
Perturbation of external neutron source can cause significant local power changes transformed into undesired safety-related events in an accelerator driven system. Therefore for the accurate design of MYRRHA sub-critical core it is important to evaluate the uncertainty of power responses caused by the uncertainties in nuclear reaction models describing the particle transport from primary proton energy down to the evaluated nuclear data table range. The calculations with a set of models resulted in quite low uncertainty on the local power caused by significant perturbation of primary neutron yield from proton interactions with lead and bismuth isotopes. The considered accidental event of prescribed proton beam shape loss causes drastic increase in local power but does not practically change the total core thermal power making this effect difficult to detect. In the same time the results demonstrate a correlation between perturbed local power responses in normal operation and misaligned beam conditions indicating that generation of covariance data for proton and neutron induced neutron multiplicities for lead and bismuth isotopes is needed to obtain reliable uncertainties for local power responses.
\end{abstract}

\section{Introduction}

The Accelerator Driven Systems (ADS), being designed to demonstrate the feasibility of efficient transmutation of Minor Actinides (MA), are conceived to couple a particle accelerator (in most projects high energy proton accelerator) and sub-critical core partially loaded with MA. The term "high energy" used hereinafter covers intermediate and high energies of particles, above the upper limit of $20-30 \mathrm{MeV}$ for neutron energies in conventional nuclear reactors. The desired transmutation rates in the sub-critical core could only be achieved with high strength of an external neutron source, which can be reached by generating neutrons in spallation reactions of protons accelerated to at least several hundred $\mathrm{MeV}$ with targets made of neutron-rich materials. The neutronic design works on such ADS projects heavily rely on model calculations, which in turn depend on the accuracy of models describing the interactions of energetic particles with matter.

In view of strengthening requirements to the reactor safety it has become common practice to support the neutronic calculations of nuclear reactors with uncertainty quantification studies. It has been shown [1-3], that the nuclear data uncertainties are most fundamental contributors to the final uncertainty of safetyrelated reactor neutronic parameters. Effective neutron multiplication factor $k_{\text {eff }}$, reactivity effects and neutron kinetic parameters remain fundamental for the sub-critical systems as well. Since these ADS safety parameters do not depend on external neutron source, the nuclear

\footnotetext{
a e-mail: astankov@sckcen.be
}

data uncertainties can be propagated [3,4] using wellestablished methods for critical reactors [5-8].

The uncertainty on external neutron source, however, may indirectly influence the reactor safety parameters as well. Source neutrons maintain fissions which determine the thermal power release in typical sub-critical systems operated at $k_{\text {eff }} \sim 0.95-0.97$. The increase in the external neutron source intensity, as well as modification of its spatial and energy distributions, could lead to an increase in the number of fissions and thus to the overheating a fuel leading to a destruction of cladding material and fuel melting. Perturbations of external neutron source are mainly driven by the perturbations of high energy part of secondary neutron spectra. The majority of modern general purpose nuclear data libraries used for particle transport calculations have energy limits $150-200 \mathrm{MeV}$. The energies of primary protons in modern ADS projects could reach $1 \mathrm{GeV}$ or even more [9] and thus the high energy tail of secondary neutron spectrum cannot be covered by nuclear data libraries. The radiation transport codes use model calculations to cover this gap from the energy of source protons down to the library range. Moreover, the evaluated nuclear data files do not contain the covariance matrices in the energy range above $20 \mathrm{MeV}$, because their calculation becomes challenging when more and more nonelastic threshold reaction channels are opening.

This work attempts to assess the uncertainties on the external source related neutronic parameters by simulating particle transport with various intranuclear cascade models describing the nuclear interactions in the energy range beyond data tables.

The objective of Work Package 11 of on-going EC FP7 Project CHANDA ("CHallenge in Nuclear DAta")

(c) The Authors, published by EDP Sciences. This is an Open Access article distributed under the terms of the Creative Commons Attribution License 4.0 (http://creativecommons.org/licenses/by/4.0/). 


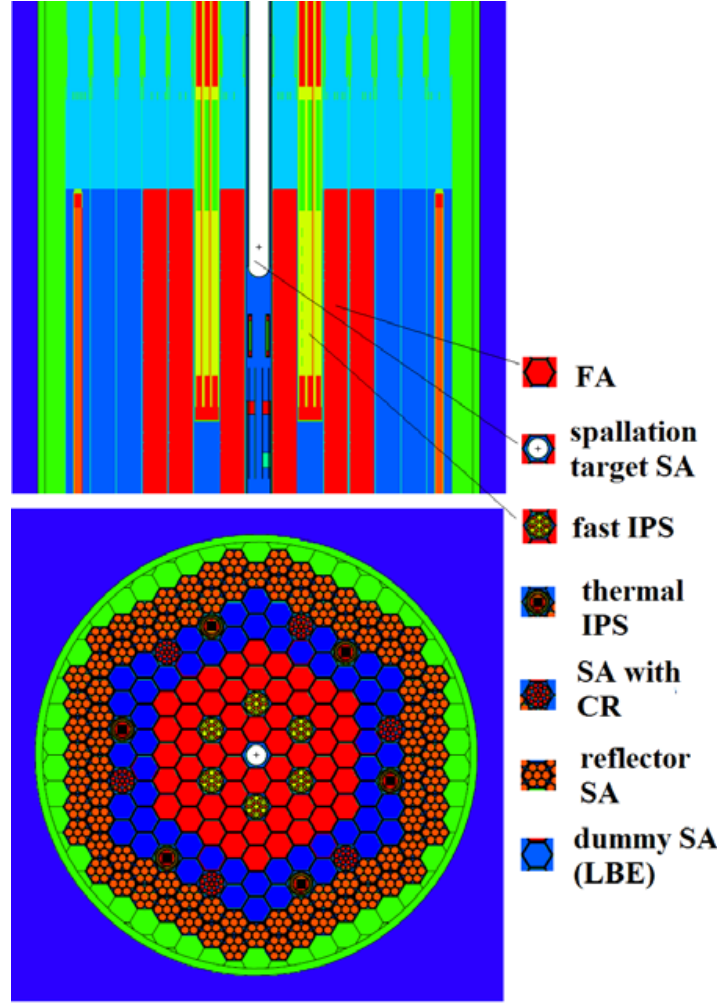

Figure 1. Vertical (top) and horizontal (bottom) cross-cuts of MYRRHA sub-critical core.

[10] is to develop a new specific methodology for uncertainty assessment of ADS safety parameters in the energy range not covered by evaluated nuclear data. The neutronic model of the Multi-purpose hYbrid Research Reactor for High-tech Applications (MYRRHA), a facility being designed at SCK•CEN, Mol, Belgium [11] has been chosen as reference and therefore it has been used in this study as well. Current detailed design is targeting a multipurpose research facility coupled with a high energy linear proton accelerator. MYRRHA will operate in both critical and sub-critical modes, in the latter case the sub-critical core is driven by spallation neutrons. Both operation modes have their specific energy and neutron flux distributions which permits a wide range of applications, from transmutation research to isotope production for medical and industrial applications. Flexible core design envisages many positions for experimental rigs to test materials for e.g. fusion reactors and new types of fuel. The core of MYRRHA is cooled by lead-bismuth eutectic (LBE), which serves also as spallation target in the sub-critical operation mode. The linear accelerator is designed to deliver up to $4 \mathrm{~mA}$ of protons accelerated to $600 \mathrm{MeV}$ that allows reaching targeted neutronic performances.

\section{The model}

A detailed reference 3D model of current MYRRHA design version 1.6 [12] has been used for particle transport calculations. Vertical and horizontal cross-cuts of the model are shown in Fig. 1.

The sub-critical core is composed of 60 fuel assemblies loaded with highly-enriched MOX fuel, In-Pile Sections (IPS) are envisaged for material irradiation in fast

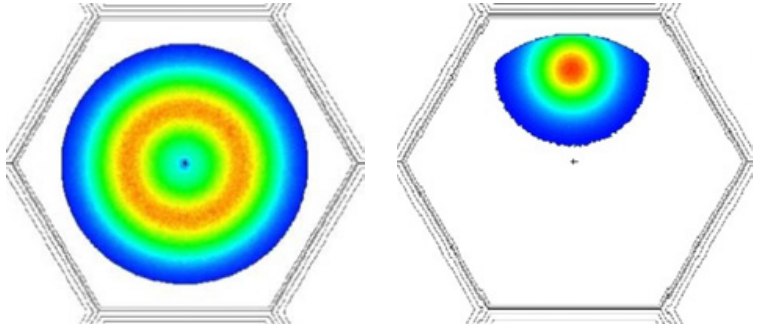

Figure 2. Footprint of reference swept Gaussian beam and offcentered non-swept Gaussian beam.

spectrum (second ring of sub-assemblies (SA), see Fig. 1) and in thermal spectrum (in the core periphery). Subassemblies with control rods, reflector assemblies loaded with beryllium oxide rods, and empty (dummy) assemblies to be loaded with fuel assemblies in the course of fuel burnup to maintain sub-criticality level, are also present in the system and can be identified in Fig. 1 .

The central SA hosts the beam tube $\sim 8 \mathrm{~cm}$ in diameter Beneath the range of protons in $\mathrm{LBE}$ of central SA, an additional irradiation volume is provided for material testing in hardest possible neutron flux spectrum. The accelerator vacuum is separated from LBE by a hemispherical $\sim 1 \mathrm{~mm}$ thin beam window made of ferriticmartensitic stainless steel of type T91. The shape of $600 \mathrm{MeV}$ proton beam is adopted to dissipate up to $2 \mathrm{MW}$ of power deposited by the beam and represents the Gaussian beam having full width at half maximum $21 \mathrm{~mm}$ swept around the central axis, sweeping radius being $21.5 \mathrm{~mm}$. The beam is deviated from horizontal direction by a set of bending magnets. The magnet closest to the target is located in the reactor hall $\sim 43 \mathrm{~m}$ above the beam window. Devices forming the necessary shape of the beam (quadrupole magnets, collimators) are also designed to be located in the reactor hall above the reactor. During the operation a loss of the prescribed shape may occur due to malfunctioning of one or simultaneously several beam focusing devices leading to the modification of external neutron distribution driving the fissions in the core. Therefore, the beam misalignment event must also be considered in the safety studies. It has been found that among various types of misalignment the loss of sweeping with the shift of the beam axis from the central axis of the tube is a crucial one [13]. The footprints of the reference and misaligned beams are shown in Fig. 2 .

As it is seen from the plot, the off-centered beam has higher intensity near the point where it hits the beam window, while the swept beam is spread along the sweeping circle. Therefore, one may expect bigger impact of the misaligned beam onto the neighbouring fuel pins, and that not only by the secondary neutrons but also by laterally straggled primary protons which undergo multiple Coulomb scattering deflecting their path from the central beam axis. The fraction of beam cut by the tube and therefore not arriving to the core does not exceed $0.5 \%$.

Particle transport simulations have been carried out with the MCNPX 2.7.0 Monte Carlo radiation transport code [14]. To model interactions above the nuclear data table range, it possess several event generators based on various combinations of intranuclear cascade, pre-equilibrium and equilibrium models. The following models and model combinations have been 
used: CEM03 [15], INCL4.5/ABLA (INAB) [16,17], INCL4/Dresner (INDr) [14,16], ISABEL/ABLA (ISAB) $[17,18]$, ISABEL/Dresner (ISDr) $[14,18]$, Bertini/ABLA (BeAB) [17,19], and Bertini/Dresner (BeDr) $[14,19]$. The discussion on their features and drawbacks can be found in [20]. In addition, the data from JENDL/HE-2007 (JHE) evaluated data library [21], both for protons and neutrons, has also been used to cover the energy range above general purpose data tables. Reference combination of data tables and models, used in SCK $\cdot$ CEN for routine design calculations, includes JEFF-3.1.2 neutroninduced evaluated data library processed at relevant temperatures [22], TENDL-2015 proton-induced library [23] and CEM03 model [15] above upper boundary of libraries $(200 \mathrm{MeV})$.

\section{Results and discussion}

\subsection{Fissions by neutrons with energies above $20 \mathrm{MeV}$}

As it has been mentioned above, the perturbation in distribution and yield of neutrons travelling from the spallation target to the core may cause corresponding perturbation of the fissions occurring in the core and energy release. Despite the angular distributions of high energy neutrons produced in a fast stage of intranuclear cascade and having energies up to the energy of primary protons are forward-peaked, a certain fraction of them, up to several percent, is present in the spectrum of neutrons travelling from the spallation target to the core. Therefore, it is interesting to estimate their contribution into the energy release in the system, in other words, how many fissions in the core they may cause.

The calculated contributions of fissions caused by neutrons with energies above $20 \mathrm{MeV}$ into the total number of fissions in the radial direction is shown in Fig. 3. The sub-assembly ring numbers begin with the inner ring surrounding the spallation target and end up with outer reflector assembly on Fig. 1.

The highest contribution of neutrons with energies above $20 \mathrm{MeV}$, however not exceeding $0.4 \%$, occurs in the first rows of fuel pins surrounding the spallation target. The fraction of high energy fissions rapidly drops with radial distance from the spallation target and slightly increases in the reflector because these neutrons are partly reflected. It has to be noted that samples containing MYRRHA MOX fuel have been placed artificially in the dummy and reflector subassemblies in order to study radial dependence of high energy fissions. Regarding the behaviour of high energy fissions among isotopes constituting MYRRHA MOX fuel, not surprisingly that ${ }^{238} \mathrm{U}$ shows the highest contributions of fissions above $20 \mathrm{MeV}$ since it fissions by fast neutrons only. Also is logical that ${ }^{241} \mathrm{Pu}$, which has highest thermal fission cross section, demonstrates the lowest fraction of high energy fissions.

Overall contribution of fissions above $20 \mathrm{MeV}$ into the total number of fissions and hence into the fission neutron multiplication in the core is below $0.01 \%$. Evaluated neutron data files for actinides contain the data up to $20-30 \mathrm{MeV}$ with rare exceptions such as TENDL library running up to $200 \mathrm{MeV}$. However, its files for actinides do not contain prompt fission neutron spectra between $20-30 \mathrm{MeV}$ and $200 \mathrm{MeV}$. The spectra

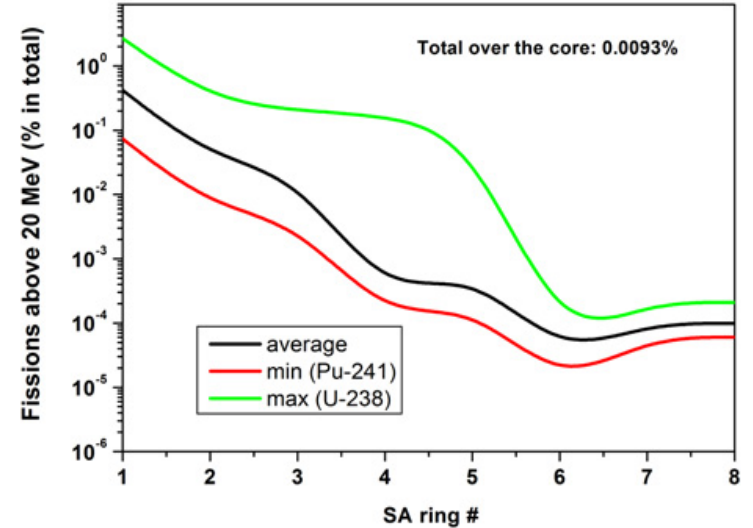

Figure 3. Distribution of fissions by neutrons with energies $>20 \mathrm{MeV}$ in the radial direction.

at $200 \mathrm{MeV}$, if provided, are the duplicates of spectra at 20-30 MeV. Therefore TENDL cannot be considered as containing evaluated data describing high energy fissions. Nevertheless, a negligible contribution of high energy fissions into the neutron balance indicates that there is no need in extending neutron induced data to higher energies for ADS applications unless some exotic projects consider spallation targets made of actinides. It can be safely concluded that the power (or energy release) response in the core to the perturbation of nuclear data/models above $20 \mathrm{MeV}$ is limited exclusively by the spallation target material, a lead-bismuth eutectic in the MYRRHA case.

\subsection{Reference beam}

The calculations have been performed in two steps to study the energy release responses. In the first step, all the particles crossing the spallation target boundary and directed to the sub-critical core have been recorded using the surface source file, a feature of MCNP code family. The particles have been transported only until this border using 7 above-mentioned physics model/data combinations and JENDL/HE library files. In the second step, this file has been used as a source for regular transport calculation in the whole model, where the particles have been transported using reference set of libraries, i.e. JEFF-3.1.2 for neutrons and TENDL-2015 for protons, complemented by CEM03 above the data table energy limits. CEM03 has also been used in the whole energy range for other heavy particles and pions. Hence the perturbation of models was limited to lead and bismuth isotopes inside the spallation target only.

The energy release in the neighbouring fuel assembly (located above the spallation target in Fig. 1) has been tallied. The mean and relative standard deviation over 8 cases have been calculated. In addition to the power factors, neutron source multiplication factor $k_{s}$ widely used in ADS neutronic studies to determine the coupling between external neutron sources and the core, has been obtained. It represents the ratio between fission neutrons and the total number of neutrons in the system [24]:

$$
k_{s}=\frac{\langle\hat{F} \Phi\rangle}{\langle\hat{A} \Phi\rangle}=\frac{\langle\hat{F} \Phi\rangle}{\langle\hat{F} \Phi\rangle+\langle S\rangle}
$$

where $\hat{F}$ and $\hat{A}$ are the fission neutron production and net neutron loss operators, $\Phi$ is the neutron flux and 
Table 1. Neutronic characteristics of reference MYRRHA sub-critical core calculated with different models /model combinations. $P_{F A}$ is the total power release in the assembly neighbouring to spallation target and $\mathrm{P}_{\mathrm{HP}}$ is the power produced in hottest part of hottest fuel pin (a fine mesh of $1 \mathrm{~cm}$ height has been used to score energy release in axial direction). The calculated $k_{\text {eff }}$ of this core is $0.95690 \pm 0.000010$.

\begin{tabular}{|l|c|c|c|c|c|c|}
\hline Model & $\mathrm{S}_{\mathrm{p}}, \mathrm{n} / \mathrm{p}$ & $\mathrm{S}, \mathrm{n} / \mathrm{p}$ & $\begin{array}{c}\mathrm{k}_{\mathrm{s}} \\
(\sigma=20 \mathrm{pcm})\end{array}$ & $\begin{array}{c}\mathrm{P}_{\text {core }}, \\
\mathrm{MW} / \mathrm{mA}\end{array}$ & $\begin{array}{c}\mathrm{P}_{\mathrm{FA}}, \\
\mathrm{kW} / \mathrm{mA}\end{array}$ & $\begin{array}{c}\mathrm{P}_{\mathrm{HP}} \\
\mathrm{kW} / \mathrm{mA}\end{array}$ \\
\hline CEM03 & 9.17 & 17.75 & 0.96392 & 34.1 & 692 & 0.126 \\
\hline INAB & 7.86 & 17.15 & 0.96389 & 35.1 & 667 & 0.117 \\
\hline INDr & 7.42 & 16.54 & 0.96354 & 32.9 & 636 & 0.113 \\
\hline ISAB & 8.36 & 17.56 & 0.96341 & 31.4 & 677 & 0.118 \\
\hline ISDr & 7.99 & 17.05 & 0.96343 & 33.2 & 655 & 0.113 \\
\hline BeAB & 8.78 & 18.12 & 0.96361 & 32.3 & 701 & 0.122 \\
\hline BeDr & 8.39 & 17.47 & 0.96359 & 34.5 & 674 & 0.117 \\
\hline JHE & 8.94 & 18.13 & 0.96422 & 33.2 & 711 & 0.126 \\
\hline Average & 8.36 & 17.45 & 0.96359 & 33.3 & 677 & 0.119 \\
\hline rsd & $7.05 \%$ & $2.96 \%$ & $45 \mathrm{pcm}$ & $3.6 \%$ & $3.65 \%$ & $4.34 \%$ \\
\hline max/min & 1.24 & 1.07 & - & 1.12 & 1.12 & 1.11 \\
\hline
\end{tabular}

$S$ is the number of neutrons source to fissions, i.e. the sum of primary source neutrons $S_{p}$ (neutrons produced from primary protons) and neutrons born in non-fission neutron multiplicative interactions $S_{n, x n}$. The core power may roughly be evaluated using $k_{s}$ [24]:

$$
P \approx I_{p} \bar{E}_{f}\left[\left(\frac{k_{s}}{1-k_{s}}\right) \frac{S}{\bar{v}}\right]
$$

Here $I_{p}$ denotes the beam current, $\bar{E}_{f}$ is the average recoverable energy released in the fission event (including the delayed energy), and $\bar{v}$ is the average number of neutrons (prompt plus delayed) per fission. This formula does not account for contribution of other neutron-induced exothermic reactions as well as other particles such as protons. Heat release in this work has been calculated directly by MCNPX and includes contributions from neutrons, protons, gammas and heavy charged particles. Therefore neutron source multiplication factor is regarded as indicative only. The neutronic parameters of reference beam are summarized in Table 1 .

The model spreads are $7 \%$ in neutron production and $4.3 \%$ in power release at hottest point, the differences between maximum and minimum values being $24 \%$ and $11 \%$, respectively. From the safety point of view these results are deemed to be quite satisfactory because, as it is seen from Table 1, the reference CEM03 model results in highest primary neutron production and second highest power release values among considered model/data combinations.

Total core power and $k_{s}$ fully correlate as expected according to Eq. (2), while it is not the case for $k_{s}$ and neutron sources, both primary and secondary. For instance, there is a significant difference between $k_{s}$ obtained with JHE and BeAB models while the source $S$ in the denominator of Eq. (1) remains practically the same. Hence the change in the number of fissions does not stem from the perturbation of the number of neutrons source to fission. Obviously the source multiplication factor $k_{s}$ in its conventional definition according to Eq. (1) does not fully account energy and angular distributions of primary neutrons, i.e. neutrons born in the interactions of primary protons with target nuclides. It can be used for rough estimates of power release in the system but definitely does not determine the sub-criticality level of the system.

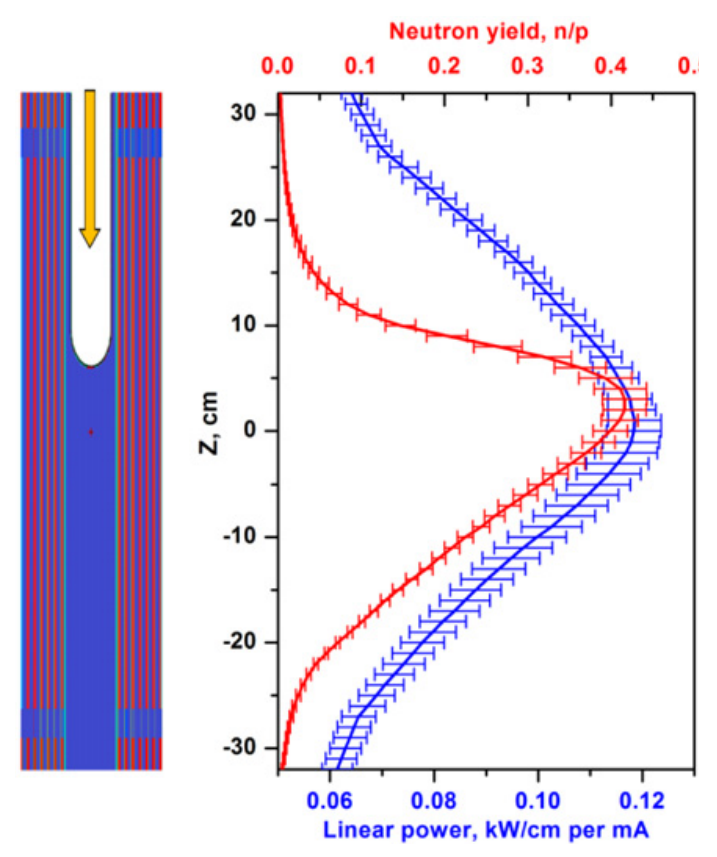

Figure 4. Neutron yield and linear power in hottest fuel pin. The sketch of spallation target sub-assembly and neighbouring fuel assemblies is shown to facilitate the comparison.

Distributions of neutron yield and power release in the hottest fuel pin are plotted in Fig. 4 along its height. It has to be noted that in the reference model the axial position of the beam window has been adjusted to center the axial power release at core mid-plane, the shift of the neutron yield being $\sim 2 \mathrm{~cm}$ upwards [12]. The plot of model average demonstrate the same shift indicating that the models have certain degree of correlation. The dominance of equilibrium neutrons in the source neutron spectrum hides possible fluctuations in the production of neutrons at the fast stage of cascade reaction. It turns out that the uncertainty in neutron yields may be reduced by tuning the model parameters describing the equilibrium stage of nuclear reactions.

The calculated neutron yields and power distributions cannot speak in favour of certain model combination even if the results of IAEA spallation model benchmark for thin targets [20] demonstrate overall better performance 
Table 2. Average over models for misaligned beam and difference from the reference case.

\begin{tabular}{|c|c|c|c|c|c|c|}
\hline Model & $\mathrm{S}_{\mathrm{p}}, \mathrm{n} / \mathrm{p}$ & $\mathrm{S}, \mathrm{n} / \mathrm{p}$ & $\begin{array}{c}\mathrm{k}_{\mathrm{s}} \\
(\sigma=20 \mathrm{pcm})\end{array}$ & $\begin{array}{c}\mathrm{P}_{\text {core }}, \\
\mathrm{MW} / \mathrm{mA}\end{array}$ & $\begin{array}{c}\mathrm{P}_{\mathrm{FA}}, \\
\mathrm{kW} / \mathrm{mA}\end{array}$ & $\begin{array}{c}\mathrm{P}_{\mathrm{HP}}, \\
\mathrm{kW} / \mathrm{mA}\end{array}$ \\
\hline Average & 8.03 & 17.66 & 0.96333 & 33.1 & 719 & 0.149 \\
\hline rsd & $7.16 \%$ & $2.92 \%$ & $55 \mathrm{pcm}$ & $3.67 \%$ & $3.61 \%$ & $4.33 \%$ \\
\hline $\begin{array}{c}\text { Difference } \\
\text { from reference } \\
\text { average }\end{array}$ & $-3.56 \%$ & $+1.2 \%$ & $-26 \mathrm{pcm}$ & $-0.6 \%$ & $+6.2 \%$ & $+25 \%$ \\
\hline
\end{tabular}
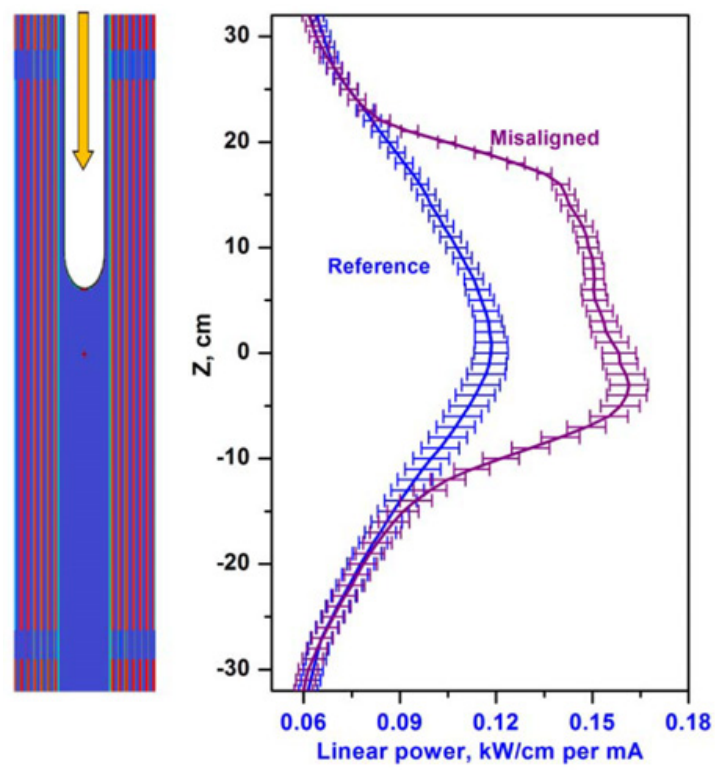

Figure 5. Axial distribution of power release in the hottest pin.

of INCL4.5 or ISABEL cascade models combined with ABLA statistical model.

\subsection{Misaligned beam}

During ADS operation, various types of malfunction may occur causing undesirable safety related events such as melting of one or several fuel pins and therefore must be quickly detected. One of such events, namely beam misalignment, has been considered here. As it was mentioned above, in order to optimize heat removal the beam must take a form of a Gaussian swept around central axis. It has been assumed that during normal operation the sweeping may be lost with simultaneous shift of the beam axis towards one of neighbouring fuel assemblies, as it is visualized in Fig. 2. The average over model/data combinations neutron sources and power factors are listed in Table 2.

The variances calculated over the models remain close to the values in Table 1 meaning that the shift does not seriously affect the relative model behaviour. The notable differences in the average neutron yield and power values are caused by the differences in the proton contribution. Indeed, a part of the misaligned beam is stopped outside the spallation target due to lateral straggling of protons. Since source neutrons have been scored in the spallation target module only, their yield decreases. Part of primary proton beam energy is deposited in the neighbouring fuel assembly drastically raising up the energy release in the hottest point. This is clearly seen from the axial power distributions plotted in Fig. 5.

Protons depositing their energy directly in or in the vicinity of the fuel pin distort the quasi-cosine shape of the axial power distribution and give rise to the energy release due to ionization losses along their path.

Neutron sources $S_{p}$ and $S_{n, x n}$ anti-correlate: while primary neutron source reduces the secondary one increases. Total core power rises only slightly while $k_{s}$, which should increase accordingly, changes in opposite direction. This is an effect of averaging.

Obviously, such type of beam misalignment event is difficult to detect by conventional power measurement. Despite part of the primary protons (about 0.5\%) is lost outside the beam tube far above the spallation target module (see Fig. 2) and the total core power is reduced accordingly (by $0.6 \%$ ), the local power seriously augments.

\section{Conclusions}

Perturbation of external neutron source can cause significant local power changes in the sub-critical core. In view of absence of evaluated neutron and proton transport data above $200 \mathrm{MeV}$, the energy gap up to $600 \mathrm{MeV}$ (that is the energy of primary protons in MYRRHA accelerator driven system) is covered in the particle transport calculations by using intranuclear cascade, preequilibrium and equilibrium model combinations. The only way to assess the uncertainties on core power responses is to perturb the model parameters or whole models. A set of model/data combinations has been used to simulate such perturbation.

It has been demonstrated that only the perturbation of neutron yield from proton and neutron interactions on lead and bismuth isotopes affects the local power in the core. Despite the spread of model predictions for primary neutron yield is rather big, the resulting uncertainties on the local power distributions are deemed to be satisfactory. However, such approach does not provide definite answer on the reliability of models which simultaneously could underestimate or overestimate neutron production.

Therefore, the extension of evaluated proton and neutron general purpose data files for $\mathrm{Pb}$ and $\mathrm{Bi}$ isotopes from 200 to $600 \mathrm{MeV}$, with this energy range covered by the data based on model calculations, is needed. The partial inclusion of covariance information, even for neutron multiplicities only, would allow assessing the uncertainty of core power responses. This would be of special importance for investigating beam perturbations such as misalignment events that are difficult to detect and which could potentially lead to safety-related problems such as fuel melting. 


\section{References}

[1] S.E. Langton, A. Buijs, J. Pencer, Ann. Nucl. En. 75, $635(2015)$

[2] R.N. Bratton, M. Avramova, K. Ivanov, Nucl. Eng. Technol. 46, 313 (2014)

[3] C.J. Diez, O. Cabellos, D. Rochman, A.J. Koning, J.S. Martinez, Ann. Nucl. En. 54, 27 (2013)

[4] P. Romojaro, et al., Ann. Nucl. En. 101, 330 (2017)

[5] D. Cacuci, Sensitivity and Uncertainty Analysis. (Chapman Hall/CRC, London, 2003)

[6] A.J. Koning and D. Rochman, J. Korean Phys. Soc. 59(23), 773 (2011)

[7] C. Díez, O. Buss, A. Hoefer, D. Porsch, O. Cabellos, Ann. Nucl. En. 77, 101 (2015)

[8] L. Fiorito, et al., Ann. Nucl. En. 101, 359 (2017)

[9] T. Sugawara, ed., Proc. Int. Symposium on Future Accelerator Driven Systems (JAEA-Review 2012043, 2012)

[10] EC Community Research and Development Information Service - CHANDA project. http:// www . cordis . europa.eu/project/rcn/110083_ en.html

[11] J. Engelen et al., Int. J. Hydrogen Energy 40, 15137 (2015)
[12] G. Van den Eynde, et al., J. Nucl. Sci. Techn. 52(7-8), 1053 (2015)

[13] Y. Celik et al., SCK•CEN-R-5980 (2015)

[14] D.B. Pelowitz, LA-CP-14-00745 (2014)

[15] S.G. Mashnik, A.J. Sierk, LA-UR-12-01364 (2012)

[16] A. Boudard et al., Phys. Rev. C 87, 014606 (2013)

[17] A. Kelic, et al., IAEA INDC(NDS)-530 (Vienna, August 2008, p. 181)

[18] Y. Yariv, IAEA INDC(NDS)-530 (Vienna, August 2008, p. 15)

[19] H.W. Bertini, Phys. Rev. 131, 1801 (1963)

[20] S. Leray et al., J. Korean Phys. Soc. 59, 791 (2011)

[21] Y. Watanabe et al., J. Korean Phys. Soc. 59(2), 1040 (2011)

[22] OECD-NEA Data Bank JEFF-3.1.2 Evaluated Data Library - Neutron data. http://www. oecdnea.org/dbforms/data/eva/evatapes/jeff 31/JEFF312

[23] A.J. Koning et al., "TENDl-2015: TALYS-based evaluated nuclear data library", https://tendl. web.psi.ch/tendl_2015/tendl2015.html

[24] H. Nifenecker, O. Meplan, S. David, Accelerator Driven Subcritical Reactors (IOP Publishing, 2010) 